\title{
An Algorithm for Automatic Measurement of KI-67 Proliferation Index in Digital Images of Breast Tissue
}

Guillermo Kemper ${ }^{*}, 1$, David Atencia ${ }^{1}$, Ivan Ortega ${ }^{1}$, Roberto Kemper ${ }^{2}$, Alejandro Yabar ${ }^{3}$

${ }^{1}$ Faculty of Engineering and Architecture, School of Electronic Engineering, University of San Martín de Porres, Lima 12, Peru.

${ }^{2}$ Institute of Pathology and Molecular Biology Arias Stella, Lima 11, Peru.

${ }^{3}$ Department of Anatomy Pathology, National Hospital Edgardo Rebagliati Martins, Lima 11, Peru.

\begin{tabular}{l} 
A R T I C L E I N F O \\
\hline Article history: \\
Received: 17 October, 2019 \\
Accepted: 24 December, 2019 \\
Online: 22 January, 2020 \\
\hline Keywords: \\
Breast Cancer \\
Image Processing, \\
Immunohistochemistry \\
KI-67 \\
\hline
\end{tabular}

\begin{abstract}
A B S T R A C T
This paper proposes an algorithm aimed at quantifying the expression of KI-67 protein in digital images of breast biopsy tissue samples obtained through an optical microscope. The algorithm allows to obtain a report on the quantity of non-proliferating and proliferating cells through the detection and quantification of KI-67. The sample analysis via software aims to reduce the level of subjectivity in the diagnosis of diseases such as breast cancer. The algorithm proposed involves the application of statistical image processing techniques, adaptive thresholds, object segmentation and color filtering. A method of analysis and quantification of overlapping cells is also proposed to improve the efficiency of the algorithm. The results of the method proposed were satisfactory, as they were highly correlated with those obtained by visual inspection by pathologists.
\end{abstract}

\section{Introduction}

Cancer is a public health problem worldwide, as evidenced by its high rates of incidence and mortality. In Peru, for example, the Lima Cancer Registry reported, in 2011 alone, about 34,000 new cases of cancer [1]. Likewise, according to the Peruvian Epidemiological Surveillance System, in the period 2006-2011, breast cancer was the second most common among Peruvian women [2], in order to choose the correct therapeutic treatment for breast cancer, guidelines often combine conventional predictors to estimate relapse/mortality risk. [3][4]

In medical research, some biomarkers have been validated for the detection and estimation of the disease progression, including breast cancer. Among them are the estrogen receptor (ER), the progesterone receptor (PR), HER2 and KI-67 [3][4]. Through a quantitative analysis, KI-67 provides a reliable estimate of the proliferative activity in a tissue. Its application to biopsies in patients with breast cancer gives doctors some evidence to treat the disease [4][5].

KI-67 is a protein that is present in all cell cycle phases (G1, S, $\mathrm{G} 2, \mathrm{M})$, except for the resting phase ( $\mathrm{G} 0)$; its presence is, therefore, quite intense and large in neoplastic proliferations [3]. For this

"Corresponding Author: Guillermo Kemper, Email: gkemperv@usmp.pe reason, the quantification of KI-67-positive cells provides valuable information to determine the magnitude of the proliferative activity.

To prove the existence of protein KI-67 in a breast biopsy tissue sample, a specific anti-protein is used with a chromogen additive allowing visualization, denominated MIB-1, this cell staining is made in a process called Immunohistochemistry (IHC). Finally, the specialist analyses the sample (in a subjective way) by eyeballing inspection through a microscope to give a report to the patient on the proliferative activity of KI-67-positive cells. According to the St. Gallen Consensus (S. Bustreo, S. OsellaAbate, P. Cassoni et al, 2016), the KI-67 index is one of the most important prognostic markers used by oncologists to determine the treatment of ER-positive breast cancer patients [6]. The number of proliferating cells in proportion to the total number of cells in a particular tissue area, called hereinafter 'proliferative index' (PI), is divided into 2 levels of prediction for Ki-67: Luminal A, if the proliferative index is less than $20 \%$; and Luminal B, if it is over or equivalent to $20 \%$ [6].

However, subjective evaluation can lead to errors in diagnosis due to several factors: room lightning, stress, fatigue, concentration, visual ability, experience, etc. They often cause that the results provided by pathologists are uncorrelated and have high 


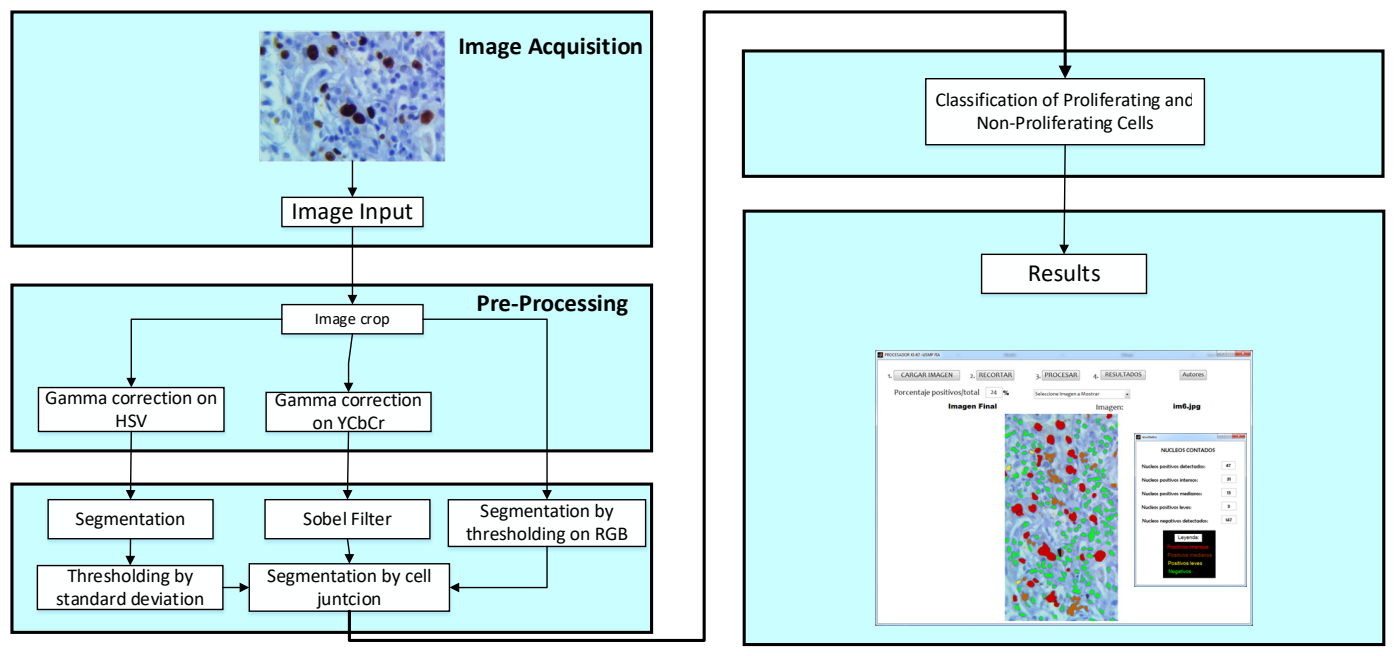

Fig. 1. Block diagram of the algorithm proposed.

variance [7][8], another factor that influences is the heterogenity of the stained sample [9]. Possible solutions to this issue have been proposed in previous research, but the main problems affecting cell detection in similar software remain: imperfection in collecting data such as noise, colour distortion and deformity in histopathological material at the stage of sample pigmentation, therefore a good calibration of the staining machine between IHC test should be made to avoid difference in pigmentation, this create a inter-laboratory variability on identical set of tumors [10].

In [11], for example, the results of the quantification algorithm were successful in analysing KI-67 with different image processing techniques such as thresholding, segmentation and labelling. However, the use of the ultra-erosion technique damaged valuable information for the evaluation of the sample.

In [12], they implement an algorithm based on mathematical description of cell morphology added with Support Vector Machine (SVM), obtaining great accordance between the digital image analysis and the "eyeballing" method.

On the other hand, M. Bouzid et al. show in [13] an interesting proposal with the use of special hardware: a multispectral camera with programmable light source. However, it increased the cost and availability of the solution. F. Kabir and N. Yusoff used in [14] the method of Random Forest (RF), consisting of predictive treelike structures, each independent of the other, that obtain their data from different random vectors, but same distribution. RF has proved to perform better than other machine learning techniques, such as neural networks, support vector machines and k-nearest neighbors. The results of the tests, however, showed an accuracy of $72 \%$ in differentiating between malignant and benign tissue.

In this paper, an alternative solution is proposed on the basis of a digital image processing algorithm designed to quantify and provide more objective results regarding the level of cell proliferation based on marker KI-67. The algorithm involves statistical image processing techniques [15], adaptive thresholds [15][16], object segmentation and colour filtering [17]. Similarly,

it proposes an overlapping cell analysis and quantification method to improve the efficiency of cell detection. These techniques have been used together to obtain satisfactory results based on the problem. The results were indeed satisfactory, as presented at the end of this document.

In the following sections, the processing stages of the algorithm proposed in this paper will be described.

\section{Description of the proposed algorithm}

The proposed algorithm involves different processing parts and stages, as shown in Fig. 1. The scheme proposed contains the stages of image acquisition, pre-processing, segmentation, classification and counting. These steps will be described below.

\subsection{Image acquisition}

Before being digitalised, the biopsy sample goes through an IHC process, which mixes the laminated sample with chemicals and special antibodies to achieve proper homogenous staining.

Currently, the procedure is performed automatically using the Ventana BenchMark XT stainer in line with the producer's manual. After staining, the sample is placed under an Olympus BX53 digital microscope with a 40x magnifying lens, 50 images were chosen to study, all were given by chief of the department of anatomy pathology of Hospital Edgardo Rebagliati Martins, all samples were chosen randomly. The images of the biopsy sample (pre-treated by IHC) are obtained from a digital camera and an optical microscope. In this case, camera resolution was $M 0=2748$ rows by $N O=3584$ columns with $40 \mathrm{x}$ zoom. The colour format of the original image (Fig. 2a) is RGB (Red, Green and Blue) called $I(x, y, z)$ with 12 bits per primary colour component (36 bits/pixel). The primary components can be expressed as $F_{R}(x, y), F_{G}(x, y)$ and $F_{B}(x, y)$.

All the parameters used in this paper were chosen taking in account the procedure mentioned previously to convert the biopsy sample into a digital image.

\subsubsection{Choosing the area of interest}

The image can be cropped to select an area of interest. This procedure grants the pathologist the freedom to choose the area to analyse according to their own criteria and preference.

The selected area will have a resolution of $M$ rows by $N$ columns (this values may vary depending on the area selected by the 
specialist). The format is also RGB with primary components $I_{R}(x, y), I_{G}(x, y)$ and $I_{B}(x, y)$.

Figs. $2 \mathrm{a}$ and $2 \mathrm{~b}$ show the original image and the cropped image, respectively.

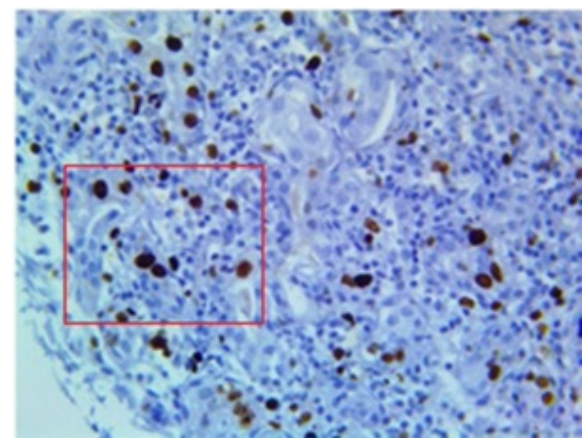

(a)

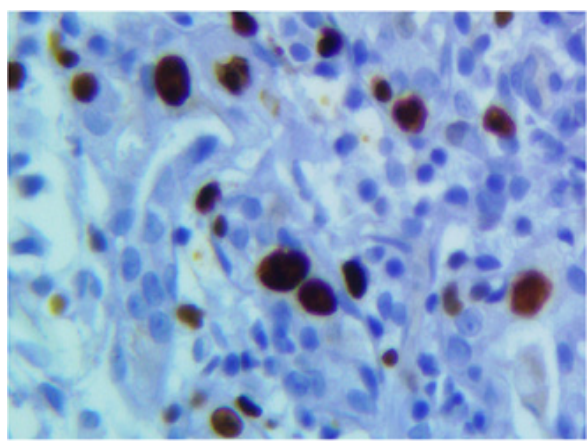

(b)

Fig. 2. (a) Original RGB image where the red square is the area to be zoomed. (b) Zoomed cropped image.

\subsection{Pre-Processing}

Pre-processing aims to improve the quality of the image obtained in order increase the efficiency of segmentation algorithms. This process involves the following image processing blocks: Gamma correction on HSV (Hue, Saturation and Value) and Gamma correction on $\mathrm{YCbCr}$ (Luminance, Chrominance Blue, Chrominance Red).

\subsubsection{Gamma correction on HSV}

The cropped image expressed in its primary components $I_{R}(x, y), I_{G}(x, y)$ e $I_{B}(x, y)$ is converted to the HSV component $I_{V}(x, y)[15]$.

Gamma correction is applied with logarithmical effect to this component so as to enhance the contrast between the background (epithelium) and the proliferating cells. This will further increase the efficiency of segmentation algorithms. The conversion applied can be expressed as [17]:

$$
I_{V}(x, y)=\max \left(I_{R}(x, y), I_{G}(x, y), I_{B}(x, y)\right)
$$

Where $\max \left(a_{1}, a_{2}, a_{3}\right)$ function generates the maximum value among $a_{1}, a_{2}$ and $a_{3}$.

The Gamma correction applied to $I_{V}(x, y)$ can be expressed as [13]:

$$
I_{V g}(x, y)=\operatorname{round}\left(\frac{I_{V}(x, y)}{255}\right)^{\gamma}
$$

where $\gamma=0.9$ and round(x) return the approximate value to the nearest integer.

Fig. 3a shows image $I(x, y)$ in RGB format; Fig. 3b shows $I_{V}(x, y)$ after applying $(1)$ to $I(x, y)$; and Fig. $3 \mathrm{c}$ shows $I_{V g}(x, y)$, where non-proliferating or blue cells obtain a colour scheme similar to that of the background, making proliferating cells stand out. Likewise, in Fig. 3c, partially proliferating cells become evident.
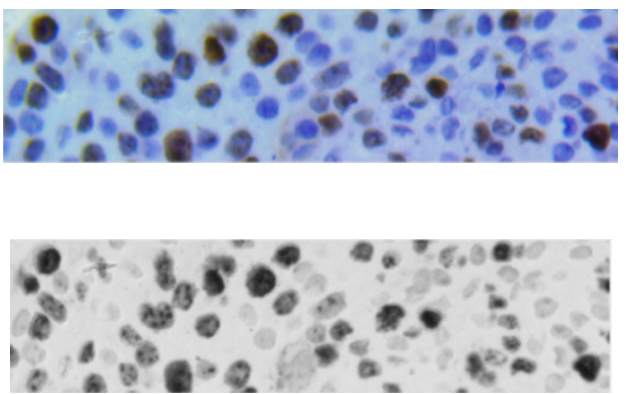

(b)

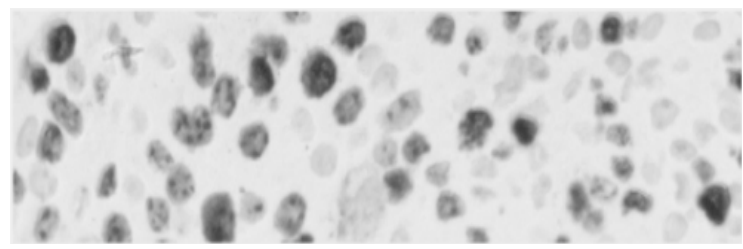

(c)

Fig. 3. Images: (a) $I(x, y)$, (b) $I_{V}(x, y)$ and (c) $I_{V g}(x, y)$

\subsubsection{Gamma correction on $\mathrm{YCbCr}$}

In parallel with the process above, the cropped image expressed in its primary components $I_{R}(x, y), I_{G}(x, y)$ and $I_{B}(x, y)$ is converted to the $\mathrm{YCbCr}$ component $I_{C b}(x, y)$ the conversion can be expressed as [15]:

$$
\begin{aligned}
I_{C b}(x, y)=128- & 37.797 I_{R}(x, y) \\
- & 74.203 I_{G}(x, y)+112 I_{B}(x, y)
\end{aligned}
$$

Gamma correction is applied to this component with $\gamma=4$ (exponential effect). This allows to displace $\mathrm{Cb}$ colour schemes from non-proliferating cells. Displacement from the original $\mathrm{Cb}$ dimensional space, shown in Fig. 4a. is shown in Fig. 4b. The image resulting from the process is expressed as $I_{C b 2}(x, y)$, as shown in Fig. 5.

The correction applied allows to separate colour schemes and improve future segmentation of the two types of cells.

\subsection{Segmentation}

In this process, objects corresponding to proliferating and nonproliferating cells are segmented. Segmentation is made from the corrected images $I_{V g}(x, y)$ and $I_{C b 2}(x, y)$ obtained from the original image (Fig. 6). 

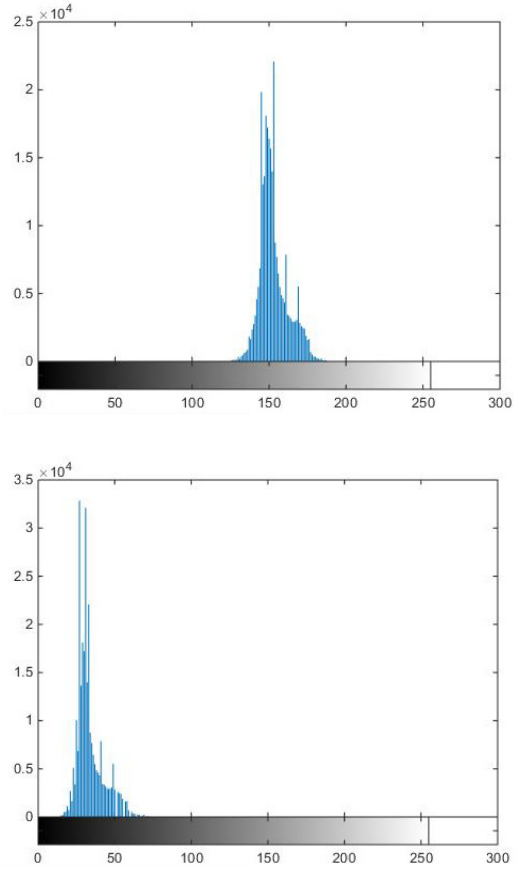

(b)

Fig. 4. (a) Original $\mathrm{Cb}$ (histogram) dimensional space. (b) Displaced Cb2 (histogram) dimensional space.

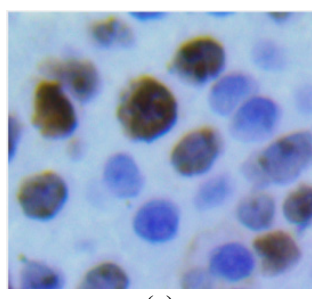

(a)

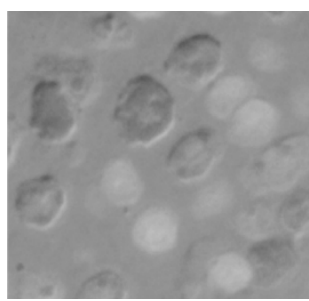

(b)

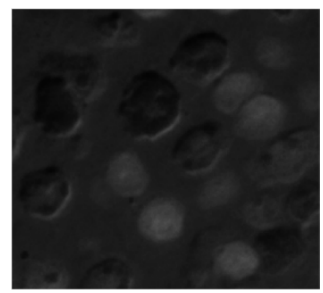

(c)

Fig. 5. (a) Original image RGB. (b) Image $I_{C b}(x, y)$. (c) Image $I_{C b 2}(x, y)$ after application of Gamma correction.

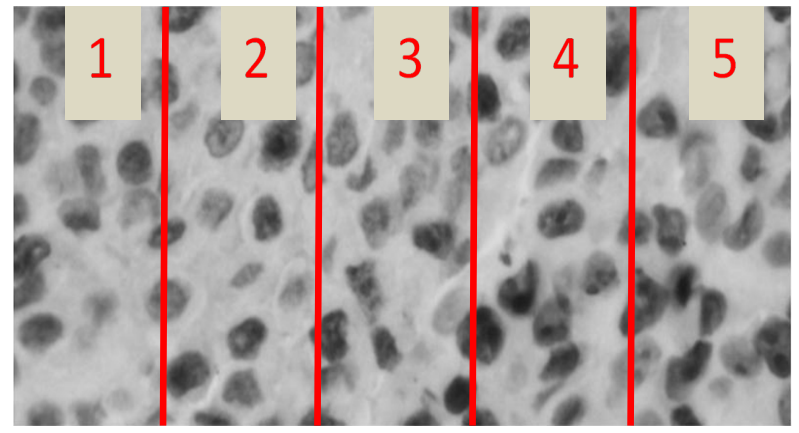

Fig. 6. Image $I_{V g}(x, y)$ to be subjected to the proliferating cell segmentation process

\subsubsection{Segmentation of proliferating cells}

To separate the background (cell tissue of no interest for diagnosis) from the proliferating cells, it was decided to use local adaptive thresholds, which result from the probability density function (obtained by histogram) and first order statistical measures (mean and variance). The steps to achieve this process are as follows:

Step 1:

Image $I_{V g}(x, y)$ is segmented in $L^{\prime}$ segments. Image segments will have a spatial resolution of $M$ rows by $\frac{N}{L^{\prime}}$ columns. The segments of image $I_{i}(x, y)$ can be expressed as:

$$
I_{i}(x, y)=I_{V g}\left(x, y+i\left(\frac{N}{L^{\prime}}\right)\right)
$$

Where $\quad x=0,1, \ldots, M-1 ; y=0,1, \ldots, \frac{N}{L^{\prime}}-1 \quad$ and $i=$ $0,1, \ldots, L^{\prime}-1$.

In this case, we have $L^{\prime}=5$, because a more even segment is obtained in terms of noise and illumination (Fig.6.).

Step 2:

The histogram of each $i$ segment is determined, which is defined as $h_{i}(r)$. In this case $r$ represents each colour scheme from 0 to 255 (Fig. 7).

Step 3:

The mean value of each segment $i$ is obtained:

$$
\bar{r}_{i}=\frac{L^{\prime}}{M \cdot N} \sum_{r=0}^{255} r h_{i}(r)
$$

Step 4:

The standard deviation for each segment $i$ is obtained:

$$
\sigma_{i}=\sqrt{\frac{L^{\prime}}{M N} \sum_{r=0}^{255}\left(r-\bar{r}_{i}\right)^{2} h_{i}(r)}
$$

Step 5:

The threshold $u_{i}$ to be used locally for each segment in order to separate cell tissue from the proliferating cell area is the result of the subtraction of the mean and standard deviation:

$$
u_{i}=\bar{r}_{i}-\sigma_{i}
$$

Step 6:

Thresholding is then applied to the segment:

$$
\hat{I}_{i}(x, y)=\left\{\begin{array}{l}
1 ; \quad I_{i}(x, y) \geq u_{i} \\
0 ; \quad I_{i}(x, y)<u_{i}
\end{array}\right.
$$

Step 7:

Finally, each segment thresholded $\hat{I}_{i}(x, y)$ is placed in the original order so as to reconstruct an image with spatial resolution of $M$ 
rows and $N$ columns, which will be called $I_{\text {pro }}(x, y)$ and will contain the proliferating cell area as shown in Fig. 8.
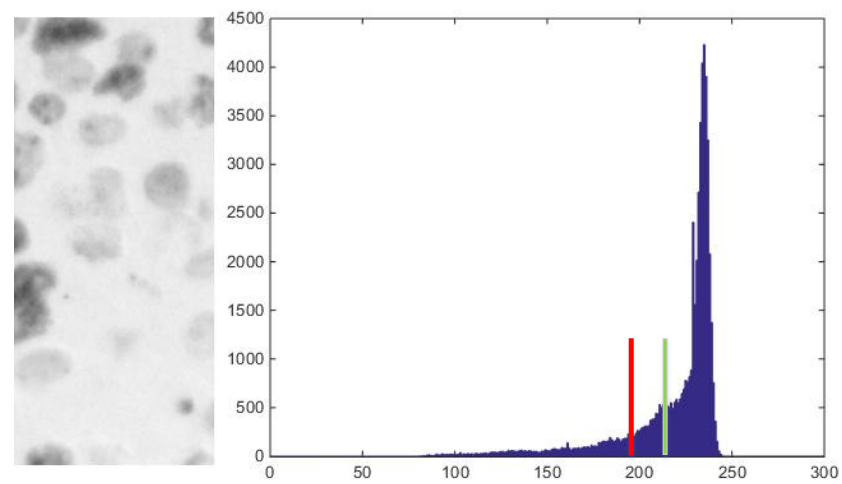

Fig. 7. Example: Segment 1 of $\left.I_{V g}(x, y)\right)$ and its histogram (in this case, the green bar indicates the mean value of the histogram and the red bar the threshold obtained).

\subsubsection{Segmentation by Sobel filter and morphology}

After identifying proliferating cells, it is necessary to spot nonproliferating cells in order to find the percentage of cells with proliferative activity in the image under evaluation $I(x, y)$.

For this process, image $I_{C b 2}(x, y)$ obtained in the step above is used. The procedure is detailed below:

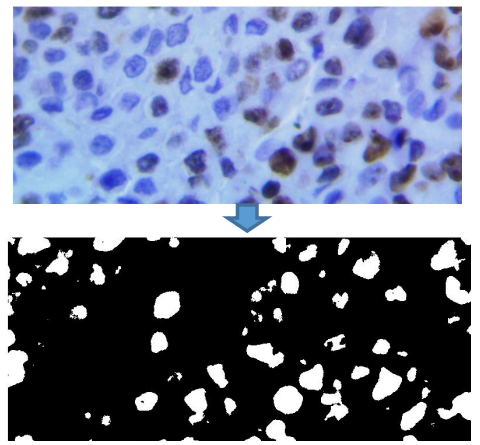

Fig. 8. Original Image and resulting binary image $I_{\text {pro }}(x, y)$ after the proliferating cell segmentation process.

Step 1:

A thresholding filter is applied to $I_{C b 2}(x, y)$ in order to segment non-proliferating cells. Thresholding can be expressed as:

$$
I_{C b 3}(x, y)=\left\{\begin{array}{c}
0 \quad ; \quad I_{C b 2}(x, y)<41 \\
I_{C b 2}(x, y) \quad ; \quad \text { other way }
\end{array}\right.
$$

The threshold was obtained from the study and evaluation of image histograms $I_{C b 2}(x, y)$ corresponding to various samples obtained. The result of the process is shown in Fig. 9.

Step 2:

Then, the Sobel filter [17] for edge detection is applied to binary images.

In this process, $I_{C b 3}(x, y)$ is convolved with each of the Sobel masks (horizontal and vertical) which are defined in matrix as [14]:

$$
\begin{gathered}
M_{v}=\left[\begin{array}{lll}
-1 & 0 & 1 \\
-2 & 0 & 2 \\
-1 & 0 & 1
\end{array}\right] \\
M_{h}=\left[\begin{array}{ccc}
-1 & -2 & -1 \\
0 & 0 & 0 \\
1 & 2 & 1
\end{array}\right]
\end{gathered}
$$

The resulting image can be expressed as (binary):

$$
I_{\text {Sobel }}(x, y)=\left\{\begin{array}{c}
1 ; \\
I_{\text {Sobel }}^{\prime}(x, y)>6 \\
0 ; \text { other way }
\end{array}\right.
$$

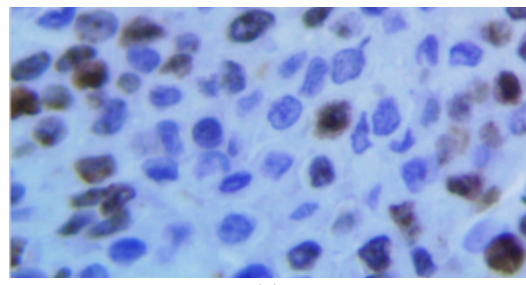

(a)

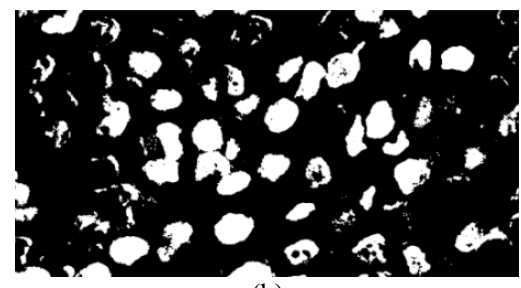

(b)

Fig. 9. (a) Original image. (b) Resulting image $I_{C b 3}(x, y)$

Where:

$$
\begin{aligned}
I_{\text {Sobel }}^{\prime}(x, y)= & \left|I_{C b 3}(x, y) * M_{h}(x, y)\right| \\
& +\left|I_{C b 3}(x, y) * M_{v}(x, y)\right|
\end{aligned}
$$

Step 3:

$I_{\text {Sobel }}(x, y)$ is subjected to a "closure" morphological operation which can be expressed through the following matrix equation:

$$
\mathbf{I}_{\text {clo }}=\left(\left[\left(\mathbf{I}_{\text {sobel }} \oplus \mathbf{B}_{v}\right) \oplus \mathbf{B}_{h}\right] \ominus \mathbf{B}_{v}\right) \ominus \mathbf{B}_{h}
$$

Where $\oplus$ indicates the dilation operation and $\ominus$ the erosion operation.

The structural elements used can be expressed as:

$$
\begin{aligned}
& B_{h}=\left[\begin{array}{lll}
1 & 1 & 1
\end{array}\right] \\
& \mathbf{B}_{v}=\left[\begin{array}{l}
1 \\
1 \\
1
\end{array}\right]
\end{aligned}
$$

In order to reform the cells detected and not to distort the visual result, gap-filling is applied to the matrix cells $\mathbf{I}_{c l o}$. This operation can be expressed as [16]: 


$$
\begin{gathered}
\mathbf{I}_{c l o}^{k}=\left(\mathbf{I}_{c l o}^{k-1} \oplus \mathbf{D}_{p}\right) \cap \dot{\mathbf{I}}_{c l o} \\
k=1,2,3, \ldots, K 0
\end{gathered}
$$

Where $k$ indicates the number of filling iteration, $\cap$ denotes the intersection operation, and $\dot{\mathbf{I}}_{c l o}$ expresses the complement of matrix $\mathbf{I}_{c l o}$. Filling is made through $K 0$ iterations until condition $\mathbf{I}_{c l o}^{k}=\mathbf{I}_{c l o}^{k-1}$ is satisfied. The structuring element $\mathbf{D}_{p}$ used can be expressed as:

$$
\mathbf{D}_{p}=\left[\begin{array}{lll}
\mathbf{0} & \mathbf{1} & \mathbf{0} \\
\mathbf{1} & \mathbf{1} & \mathbf{1} \\
\mathbf{0} & \mathbf{1} & \mathbf{0}
\end{array}\right]
$$

Finally, the result of this stage is show on Fig.10 and placed in matrix $\mathbf{I}_{\text {Sobelf }}$ :

$$
\mathbf{I}_{\text {Sobelf }}=\mathbf{I}_{\text {clo }}^{K 0}
$$

\subsubsection{Segmentation by thresholding on primary components}

At this stage, thresholding is applied to the primary RGB components to separate proliferating and non-proliferating cells from the epithelium (background). The thresholds used were obtained from experimental tests performed with images from different tissue samples. The procedure helps remove the area of the epithelium and highlight cells of interest. The steps of the procedure are as follows:

Step 1:

The primary components of the original image $\mathbf{I}$ are thresholded (Fig 11a):

$$
Z_{R}(x, y)=\left\{\begin{array}{lc}
0, & I_{R}(x, y)<128 \\
1, & I_{R}(x, y) \geq 128 \vee I_{G}(x, y) \geq 128
\end{array}\right.
$$

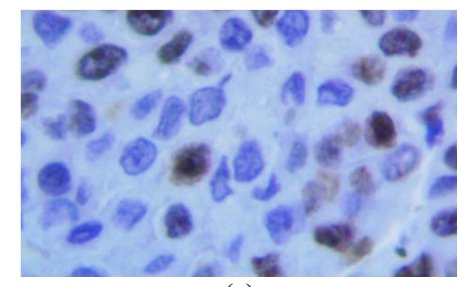

(a)

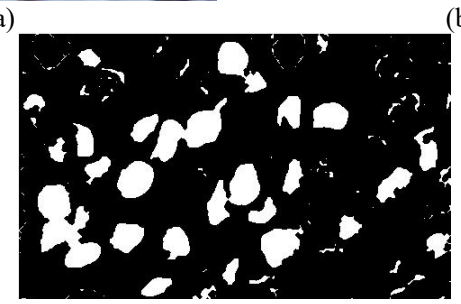

(c)

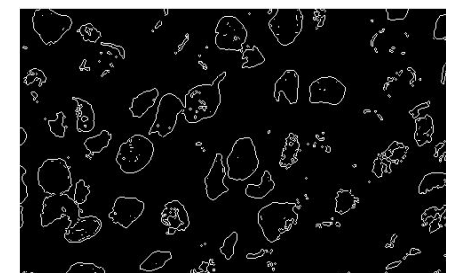

(b)

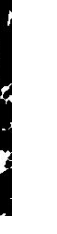

Fig. 10. (a) Cropped original image. (b)Resulting Image after applying Sobel Filter. (c)Final Image $I_{\text {Sobel }}(x, y)$ after gap-filling.

$$
Z_{G}(x, y)=\left\{\begin{array}{cc}
0, & I_{G}(x, y)<128 \\
1, & \text { other way }
\end{array}\right.
$$

$$
Z_{B}(x, y)=\left\{\begin{array}{lc}
0, & I_{B}(x, y)<128 \\
1, & I_{B}(x, y) \geq 128 \vee I_{G}(x, y) \geq 128
\end{array}\right.
$$

Step 2:

A matrix of zeros $I_{c e l}(x, y)$ with spatial resolution $M \times N$ is generated. Then some pixels of $I_{c e l}(x, y)$ are changed to 1 from the following condition:

$$
I_{c e l}(x, y)=\left\{\begin{array}{cc}
1, & \left(Z_{R}(x, y)=1\right) \wedge \\
& \left(Z_{G}(x, y)=1\right) \wedge \\
& \left(Z_{B}(x, y)=1\right) \\
0, & \text { other way }
\end{array}\right.
$$

$I_{c e l}(x, y)$ finally constitutes a template where cell pixels have value 1 (Fig. 11b).

Step 3:

Finally, the matrix of non-proliferating cells (Fig. 11c) is obtained from the following expression:

$$
I_{\text {nopro }}(x, y)=\left\{\begin{array}{cc}
1, & \left(I_{\text {cel }}(x, y)=1\right) \vee \\
( & \left.I_{\text {sobelf }}(x, y)=1\right) \wedge \\
& \left(I_{\text {Pro }}(x, y) \neq 1\right) \\
0, & \text { other way }
\end{array}\right.
$$

\subsubsection{Segmentation by cell junction}

The combination of the proliferating and non-proliferating cell areas results in a binary image containing two types of segmented cells. This image is obtained as follows:

$$
I_{\text {total }}(x, y)=\left\{\begin{array}{lc}
1, & \left(I_{\text {pro }}(x, y)=1\right) \vee \\
0, & \left(I_{\text {nopro }}(x, y)=1\right) \\
\text { other way }
\end{array}\right.
$$

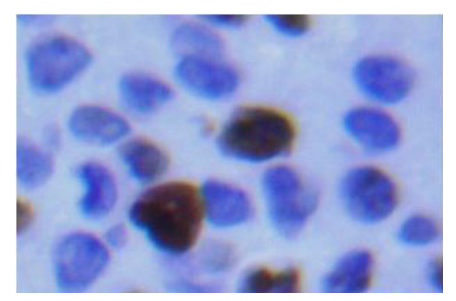

(a)

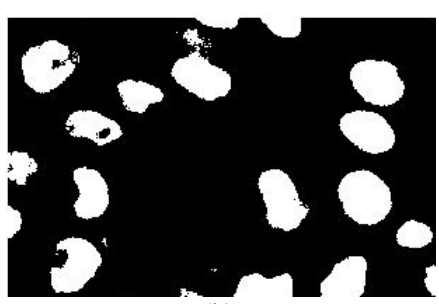

(b)

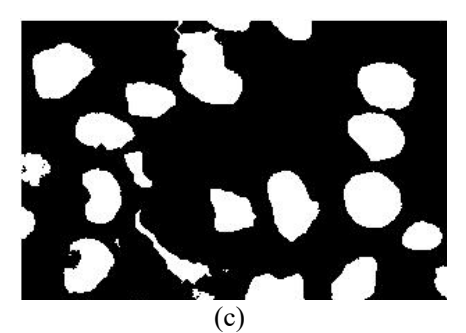

Fig. 11. (a) Cropped original image. (b) Image $I_{c e l}(x, y)$. (c) Image $I_{\text {nopro }}(x, y)$. 
However, one of the problems still present when the cells detected are counted is that some objects are made up of two or more joint or overlapped cells, which results in the algorithm giving a wrong diagnosis. In that sense, as in an acquisition with $40 \mathrm{x}$ magnification the average size of a cell is between 200 and 1500 pixels, we can consider that an object of $I_{\text {total }}(x, y)$ with a size above 1500 pixels is composed of more than one cell. This will definitely affect the counting, as an object is in principle composed of a single cell. To solve the problem, the following cell separation algorithm is proposed in order to minimise the counting error:

\section{Step 1:}

Consider $I_{j}\left(x_{j}, y_{j}\right)$ a binary segment of $I_{\text {total }}(x, y)$ that contains object $j$ with more than 1500 pixels. In this case, $x_{j}=$ $0,1,2 \ldots, m_{j}-1 \ldots$, and $y_{j}=1,2, \ldots, n_{j}-1$; i.e. the segment size is $m_{j} \times n_{j}$ pixels.

\section{Step 2:}

We perform image rotation for different angles $\theta\left(\theta_{0}=0^{\circ}, \theta_{1}=\right.$ $15^{\circ}, \theta_{2}=30^{\circ}, \theta_{3}=60^{\circ}, \theta_{4}=75^{\circ}$ and $\left.\theta_{5}=90^{\circ}\right)$. In this case, the rotation axis passes through the centre of image $I_{j}\left(x_{j}, y_{j}\right)$. The range of $15^{\circ}$ between rotation angles was chosen based on the best results obtained in various tests of cell separation. Each turn generates an image that can be expressed as $I_{j}^{\theta}\left(x_{j}^{\prime}, y_{j}^{\prime}\right)$, where $\theta$ indicates the rotation angle. The spatial dimensions of each $I_{j}^{\theta}\left(x^{\prime}{ }_{j}, y^{\prime}{ }_{j}\right)$ is expressed as $m_{j}^{\theta}$ rows by $n_{j}^{\theta}$ columns.

Step 3:

The horizontal projection $I_{j}^{\theta}\left(x^{\prime}{ }_{j}, y^{\prime}{ }_{j}\right)$ is obtained:

$$
P_{j}^{\theta}\left(y_{j}^{\prime}\right)=\sum_{x^{\prime}{ }_{j}=0}^{m_{j}^{\theta}-1} I_{j}^{\theta}\left(x_{j}^{\prime}, y_{j}^{\prime}\right)
$$

Step 4:

It is important to assess the central area of protection for each $P_{j}^{\theta}\left(y^{\prime}{ }_{j}\right)$, which is why the furthest areas of the vector are deleted:

$$
\hat{P}_{j}^{\theta}\left(y_{j}^{\prime}\right)=\left\{\begin{array}{cc}
P_{j}^{\theta}\left(y^{\prime}{ }_{j}\right), & \left(y^{\prime}{ }_{j}>q_{0} \cdot n_{j}^{\theta}\right) \vee \\
0, & \left(y_{j}^{\prime}<q_{1} \cdot n_{j}^{\theta}\right) \\
0 & \text { other way }
\end{array}\right.
$$

Where $q_{0}=0.3$ and $q_{1}=0.7$

Step 5:

Value $\hat{P}_{j}^{\theta}\left(y_{0}(\theta, j)\right)$ is obtained, which is defined as the minimum value of the projection $\hat{P}_{j}^{\theta}\left(y^{\prime}{ }_{j}\right)$. In this case, $y_{0}(\theta, j)$ is the position where $\hat{P}_{j}^{\theta}\left(y^{\prime}{ }_{j}\right)$ is the minimum value.

Step 6:

The $\mathbf{W}_{\mathrm{j}}$ vector is then obtained:

$$
\mathbf{W}_{\mathrm{j}}=\left[\begin{array}{c}
\hat{P}_{j}^{\theta_{0}}\left(y_{0}\left(\theta_{0}, j\right)\right) \\
\hat{P}_{j}^{\theta_{1}}\left(y_{0}\left(\theta_{1}, j\right)\right) \\
\vdots \\
\hat{P}_{j}^{\theta_{5}}\left(y_{0}\left(\theta_{5}, j\right)\right)
\end{array}\right]
$$

Step 7:

The minimum value of vector $\mathbf{W}_{\mathrm{j}}$ is obtained, which is defined as $\hat{P}_{j}^{\theta_{L 0}}\left(y_{0}\left(\theta_{\mathrm{L} 0}, j\right)\right)$, where $\theta_{L 0}$ is the angle corresponding to the minimum value of $\mathbf{W}_{\mathrm{j}}$.

Step 8:

Then $Z_{j}^{\prime}\left(x_{j}^{\prime}, y^{\prime}{ }_{j}\right)$ is obtained from $I_{j}^{\Theta_{L 0}}\left(x_{j}^{\prime}, y_{j}^{\prime}\right)$ :

$$
Z_{j}^{\prime}\left(x_{j}^{\prime}, y^{\prime}{ }_{j}\right)=\left\{\begin{array}{c}
0 \quad ; \quad y_{j}^{\prime}=y_{0}\left(\theta_{L 0}, j\right) \\
I_{j}^{\theta_{L 0}}\left(x_{j}^{\prime}, y_{j}^{\prime}\right) ; \text { other way }
\end{array}\right.
$$

Step 9:

Rotation of $-\theta_{\mathrm{L} 0}$ over $I_{j}^{\theta_{\mathrm{L} 0}}\left(x^{\prime}{ }_{j}, y^{\prime}{ }_{j}\right)$ is applied to restore the original orientation (although distortion is present due to the interpolation applied to the rotation process). After rotation, image $V_{j}\left(x_{j}^{\prime}, y_{j}^{\prime}\right)$ is obtained.

Step 10:

Finally, the block of image $V_{j}\left(x^{\prime}, y^{\prime}{ }_{j}\right)$ is inserted in $I_{\text {total }}(x, y)$ (in the region where $I_{j}\left(x_{j}, y_{j}\right)$ was extracted).

$$
I_{\text {total }}\left(x a_{j}+x_{j}^{\prime}, y a_{j}+y_{j}^{\prime}\right)=V_{j}\left(x_{j}^{\prime}, y_{j}^{\prime}\right)
$$

Where $x a_{j}$ and $y a_{j}$ are the coordinates of the first pixel segment extracted $I_{j}\left(x_{j}, y_{j}\right)$.

Fig. 12a shows original areas of $I_{\text {total }}(x, y)$ containing objects made up of more than one cell. Fig. 12b shows the results obtained after applying the algorithm of cell separation.

\subsection{Classification and counting of proliferating and non- proliferating cells}

From the total number of cells detected in image $I_{\text {total }}(x, y)$, we proceed to classify and count proliferating and non-proliferating cells.

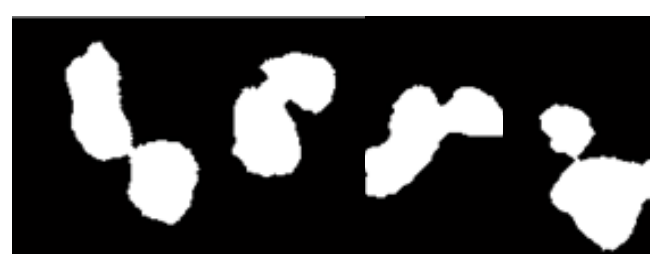

(a)

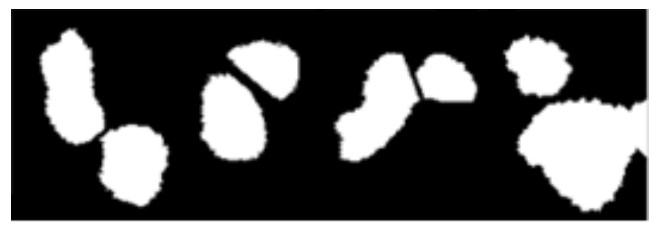

(b)

Fig. 12. (a) Samples with junction. (b) Samples after the separation process. 
Cooperation with pathologists was managed so that the parameters of cell classification were correct and a good diagnosis could be achieved. In this case, doctors requested to classify proliferating cells into three sub groups: strongly proliferating, moderately proliferating and poorly proliferating. The classification criterion is based on the size of the cell area affected by the KI-67 protein.

Image $I_{\text {total }}(x, y)$ is subjected to a labelling algorithm [16] that will label each cell detected as a $k$ object. In this case, $k$ is the object counter detected in the image. For a total of $Q$ objects, we have $k=0,1,2, \ldots Q-1$.

\subsubsection{Classification of Proliferating and Non-Proliferating Cells}

For the classification stage, counters Total ${ }_{\text {pro }}=0$ (proliferating cell counter) and counter Total $_{\text {npro }}=0$ (non-proliferating cell counter) are initialised.

$\mathrm{T}_{\text {obj }}$ is defined as the total number of objects or cells contained in $\mathrm{I}_{\text {total }}(\mathrm{x}, \mathrm{y})$. In this context, $\mathrm{k}$ is taken to be the object or cell number to be processed. In this case $\mathrm{k}=0,1, \ldots, \mathrm{T}_{\mathrm{obj}}-1$. We make $\mathrm{k}=0$ and carry out the following procedure:

\section{Step 1:}

A segment $I_{k}\left(x^{*}, y^{*}\right)$ of image $I_{\text {total }}(x, y)$ is extracted consisting of $M_{k}$ rows and $N_{k}$ columns, where $x^{*}=0,1, \ldots, M_{k}-1$ and $y^{*}=0,1, \ldots, N_{k}-1$. In this case, $I_{k}\left(x^{*}, y^{*}\right)$ is the image segment containing the object $k$ detected in $I_{\text {total }}(x, y)$.

Step 2:

A flag $f_{k}$ is updated through the following expression:

$$
f_{k}=\sum_{x^{*}=0}^{M_{k}-1} \sum_{y^{*}=0}^{N_{k}-1} I_{k}\left(x^{*}, y^{*}\right) \cdot I_{\text {pro }}\left(x_{0_{k}}+x^{*}, y_{0_{k}}+y^{*}\right)
$$

Where $x_{0_{k}}$ and $y_{0_{k}}$ are the coordinates of the first pixel of $I_{k}\left(x^{*}, y^{*}\right)$ in $I_{\text {total }}(x, y)$.

Step 3:

If $f_{k} \neq 0$, the cell under evaluation is considered proliferating and the counter Total pro increases by one unit. Otherwise, the counter Total $_{\text {npro }}$ increases. Within the requirements of doctors, a sub classification of proliferating cells was made in 3 levels of proliferation: strongly proliferating, moderately proliferating and poorly proliferating.

In this way, when $f_{k} \neq 0$, steps 4 to 9 are executed.

Step 4:

A temporary matrix $C_{k}\left(x^{*}, y^{*}\right)$ consisting of $M_{k}$ rows and $N_{k}$ columns is generated and applied:

$$
C_{k}\left(x^{*}, y^{*}\right)=\left\{\begin{array}{c}
1 ; \text { si } I_{\text {pro }}\left(x_{0_{k}}+x^{*}, y_{0_{k}}+y^{*}\right) \neq 0 \\
0 ; \quad \text { other way }
\end{array}\right.
$$

We count each pixel belonging to an area that reacted to KI-67:

$$
\operatorname{Apro}_{k}=\sum_{x^{*}=0}^{M_{k}-1} \sum_{y^{*}=0}^{N_{k}-1} C_{k}\left(x^{*}, y^{*}\right)
$$

Step 6:

Similarly, for the non-proliferating cell area, a matrix $C^{\prime}{ }_{k}\left(x^{*}, y^{*}\right)$ consisting of $M_{k}$ rows and $N_{\mathrm{k}}$ columns is generated and applied:

$$
C^{\prime}{ }_{k}\left(x^{*}, y^{*}\right)=\left\{\begin{array}{lr}
1, I_{\text {nopro }}\left(x_{0_{k}}+x^{*}, y_{0_{k}}+y^{*}\right) \neq 0 \\
0, & \text { other way }
\end{array}\right.
$$

Step 7:

The pixels of the regions that did not react to the protein KI-67 are counted:

$$
\operatorname{Anpro}_{k}=\sum_{x^{*}=0}^{M_{k}-1} \sum_{y^{*}=0}^{N_{k}-1} C^{\prime}{ }_{k}\left(x^{*}, y^{*}\right)
$$

Step 8:

Then the intensity factor of each cell is found:

$$
R_{k}=\frac{\text { Apro }_{k}}{\text { Anpro }_{k}+\text { Apro }_{k}}
$$

Step 9:

Flags of proliferation $f p_{k}, f m_{k}$ and $f d_{k}$ are updated:

$$
f p_{k}=\left\{\begin{array}{l}
1, \quad R_{k} \geq 0.7 \\
0, \text { other way }
\end{array}\right.
$$

$$
\begin{gathered}
f m_{k}=\left\{\begin{array}{lr}
1, & R_{k}>0.2 \wedge R_{k}<0.7 \\
0, & \text { other way }
\end{array}\right. \\
f d_{k}= \begin{cases}1, & R_{k} \leq 0.2 \\
0, & \text { other way }\end{cases}
\end{gathered}
$$

For each $k$ where $f_{k} \neq 0$ there is only one active flag $\left(f p_{k}, f m_{k}\right.$ and $\left.f d_{k}\right)$.

Three counters (initialised to zero), defined as strong, medium and weak, are updated. They are updated as follows: if $f p_{k}=1$, the strong counter is incremented by one; if $\mathrm{fm}_{k}=1$, the medium counter is increased by one; if $f d_{k}=1$, the weak counter is increased by one. These counters store the amount of subclassified cells. Note that strong + medium + weak $=$ Total $_{\text {pro }}$. This data is granted to the pathologist for evaluation and diagnosis, as shown in Table 1 .

Then, $k$ increases by one unit and we go back to step 1 . The procedure ends when $k=T_{o b j}$.

Step 10:

After evaluating all the cells, we find the percentage of proliferating cells and obtain:

$$
\% \text { Diagnosis }=\left(\frac{\text { Total }_{\text {pro }}}{\text { Total }_{\text {pro }}+\text { Total }_{\text {npro }}}\right) \times 100
$$

Step 5: 
Table 1. Results after test.

\begin{tabular}{ccc}
\hline Total counted nucleus & Quantity & Percentage (\%) \\
\hline Negative nucleus & 94 & 88 \\
\hline Positive nucleus & 13 & 12 \\
\hline Total & 107 & 100 \\
\hline Positive counted nucleus & & \\
\hline Strong positive nucleus & 2 & 15 \\
\hline Medium positive nucleus & 7 & 54 \\
\hline Weak Positive Nucleus & 4 & 31 \\
\hline
\end{tabular}

\section{Results}

To prove that the software can be used by specialists, the results obtained by it had to be validated against the results provided by doctors; they were requested to make the same analysis on equal samples. The doctors that carried out the tests are specialised in pathology and work in different institutions in the city of Lima.

Table 2, 3, 4, 5 and 6 show the results obtained in different cases. When comparing the results obtained by the application against the diagnosis given by doctors, it was concluded that, in samples with a clear KI-67-active cell rate (either in a very small or very high percentage in proportion to the total number of cells), doctors using the eyeballing technique obtain results very similar to those of the application. But when samples present some difficulty at the time of counting, it is observed that the results among doctors are poorly correlated with each other and only a few can give a diagnosis similar to that of the application. This is because doctors are subject to different stimuli that influence their performance and they have different counting methods, such as hotspots, random areas, or groups near adjacent hotspots [7], while the software will always proceed in the same way.

In Figure 13 it is shown a section of an evaluated image by both the medical specialist and the developed algorithm. Five cells have been enumerated and marked in a rectangle, named: cell 1 , cell 2 , cell 3 , cell 4 and cell 5 .

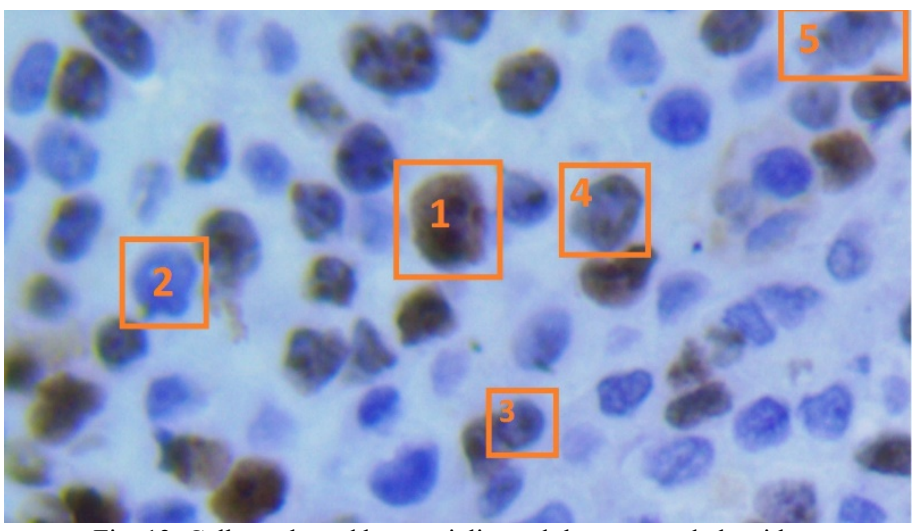

Fig. 13. Cells evaluated by specialist and the proposed algorithm.

For cell 1, both medical specialist and proposed algorithm confirmed that represent a proliferative cell, meaning it was stained in brown color by the IHC process.

For cell 2, again both specialist and algorithm concurred that the cell was non-proliferative, stained with a blue tone by the IHC process. In case of cell 3 and 5, the staining was lower than cell 1, specialist marked the cell as non-proliferative and the algorithm marked as proliferative, is in this scenario in which the subjectivity of human eye turn the proliferation index and can occur divergence in the diagnosis. In case of cell 4 , shows partial staining and both specialist and algorithm matched as proliferative.

To demonstrate the error trend, the table below shows the calculus of coincidence between the software and the specialists, and its measuring using the Kappa coefficient.

Defining Kappa coefficient as [18]:

$$
\widetilde{K}=\frac{\bar{T}-\bar{T}_{e}}{1-\bar{T}_{e}}
$$

Define $\bar{T}$ as:

$$
\bar{T}=\frac{1}{N_{0}} \sum_{i=1}^{N_{0}} \beta_{i}
$$

Where $N_{0}$ is the total number of category, in this case two categories $N_{0}=2$ : Luminal A, and Luminal B, as explained on [6], Luminal A if diagnosis was $<20 \%$ and Luminal B if was $\geq 20 \%$.

$\beta_{i}$ is defined as:

$$
\beta_{i}=\frac{1}{\alpha(\alpha-1)}\left[\left(\sum_{j=1}^{N_{0}} \alpha_{i j}^{2}\right)-\alpha\right]
$$

Where $\alpha$ is the number of occurrences from a category, $\alpha_{i j}$ is the number of times the specialist chose a diagnosis in the category $i$ and the software chose category $j$.

Finally:

$$
\bar{T}_{e}=\sum_{j=1}^{N_{0}} \varepsilon_{j}^{2}
$$

Where $\varepsilon_{j}$ is calculated using the following expression:

$$
\varepsilon_{j}=\frac{1}{N_{0} \alpha}\left(\sum_{i=1}^{N_{0}} \alpha_{i j}\right)
$$

To calculate precision and recall of specialist vs software, $F-$ score value was calculated [19]:

$$
F-\text { score }=\left(\frac{\left(\rho^{2}+1\right) \cdot \text { precision } \times \text { recall }}{\rho^{2} \cdot \text { precision } \times \text { recall }}\right)
$$

Where precision and recall were calculated as:

$$
\text { precision }=\frac{w p}{w p+l p}
$$


And:

$$
\text { recall }=\frac{w p}{w p+\ln }
$$

Where:

$w p$ : true positive, a cell that specialist said was proliferative and algorithm said was proliferative.

$l p$ : false positive, a cell that specialist said wasn't proliferative and algorithm said it was proliferative.

$\ln$ : false negative, a cell that said specialist said was proliferative but algorithm said wasn't proliferative.

$F-$ score is considered balanced so:

$$
\rho=1
$$

Finally, $F-$ score is calculated as:

$$
F-\text { score }=\frac{2 w p}{(2 w p+l p+l n)}
$$

The average result for F-score of the samples was of 0.8764 , showing a high concordance between the software and specialist. The software detects a greater number of cells than the specialist, so the F-score is not perfect, however, these cells not accounted were mostly discarded by specialist errors.

As shown in the Tables 2, 3, 4, 5 and 6 there is a remarkable variation among specialists in the Luminal $\mathrm{B}$ diagnosis made by counting. The poor agreement in the diagnosis is due to the difference between stained and non-stained nuclei as rapidly observed by the specialist. Nevertheless, two specialists gave the same result as the software; it should be noted that a proliferation index $>20 \%$ is needed to share the same diagnosis.

Tables 7, 8 and 9 show the difference among specialists when giving their diagnosis. The Kappa index among specialists is not constant, an average of 0.6863 , which demonstrates, once again, the difference generated by the subjective analysis by eyeballing [9][10].

Table 2. Comparison Software vs. Specialist 1

$$
\text { Specialist 1: Kappa } \widetilde{K}=0.689
$$

\begin{tabular}{cccc}
\hline Specialist 1 & Luminal A & Luminal B & TOTAL \\
Software & & & \\
\hline Luminal A & 7 & 3 & 10 \\
\hline Luminal B & 0 & 9 & 9 \\
\hline TOTAL & 7 & 12 & 19 \\
\hline
\end{tabular}

Table 3. Comparison Software vs. Specialist 2 Specialist 2: Kappa $\widetilde{K}=0.689$

\begin{tabular}{cccc}
\hline Specialist 2 & Luminal A & Luminal B & TOTAL \\
\hline Luftware & & & \\
\hline Luminal A & 7 & 3 & 10 \\
\hline TOTAL & 7 & 12 & 9 \\
\hline
\end{tabular}

Table 4. Comparison Software vs. Specialist 3 Specialist 3: Kappa $\widetilde{K}=1$

\begin{tabular}{cccc}
\hline Specialist 3 & Luminal A & Luminal B & TOTAL \\
Software & & & \\
\hline Luminal A & 10 & 0 & 10 \\
\hline Luminal B & 0 & 9 & 9 \\
\hline TOTAL & 0 & 9 & 19 \\
\hline
\end{tabular}

Table 5. Comparison Software vs. Specialist 4 Specialist 4: Kappa $\widetilde{K}=1$

\begin{tabular}{cccc}
\hline Specialist 4 & Luminal A & Luminal B & TOTAL \\
\hline Luminal A & 10 & 0 & 10 \\
\hline Luminal B & 0 & 9 & 9 \\
\hline TOTAL & 0 & 9 & 19 \\
\hline
\end{tabular}

Table 6. Comparison Software vs. Specialist 5

Specialist 5: Kappa $\widetilde{K}=0.787$

\begin{tabular}{cccc}
\hline Software & Luminal A & Luminal B & TOTAL \\
\hline Luminal A & 10 & 0 & 10 \\
\hline Luminal B & 2 & 7 & 9 \\
\hline TOTAL & 12 & 7 & 19 \\
\hline
\end{tabular}

Table 7. Comparison Specialist 1 vs. Specialist 2

Specialist 1 vs Specialist 2: Kappa $\widetilde{K}=0.774$

\begin{tabular}{cccc}
\hline Specialist 1 & & & \\
Specialist 2 & Luminal A & Luminal B & TOTAL \\
\hline Luminal A & 6 & 1 & 7 \\
\hline Luminal B & 1 & 11 & 12 \\
\hline TOTAL & 7 & 12 & 19 \\
\hline
\end{tabular}

Table 8. Comparison Specialist 2 vs. Specialist 4

Specialist 2 vs Specialist 4: Kappa $\widetilde{K}=0.596$

\begin{tabular}{cccc}
\hline Specialist 2 & & & \\
Specialist 4 & Luminal A & Luminal B & TOTAL \\
\hline Luminal A & 7 & 0 & 7 \\
\hline Luminal B & 4 & 8 & 12 \\
\hline TOTAL & 11 & 8 & 19 \\
\hline
\end{tabular}

Table 9. Comparison Specialist 1 vs. Specialist 5

Specialist 1 vs Specialist 5: Kappa $\widetilde{K}=0.689$

Specialist 1 Luminal A Luminal B TOTAL

Specialist 5

\begin{tabular}{cccc}
\hline Luminal A & 7 & 3 & 10 \\
\hline Luminal B & 0 & 9 & 9 \\
\hline TOTAL & 7 & 12 & 19 \\
\hline
\end{tabular}




\section{Conclusions}

In this publication, an algorithm to quantify the proliferative index of the KI-67 marker in histological samples of breast tissue has been developed that proves to give results more reliable than results given by specialist, thanks to image processing techniques, this algorithm can help to provide a better diagnostic to treat breast cancer when applied the result with other biomarkers to diagnose cancer.

A software application was developed. It meets the standards and criteria that pathologists use for the evaluation of the immunohistochemistry method and it is approved by specialists, as they consider that, when testing the software in their work centres, results are reliable.

The iterative algorithm of cell separation is computationally heavy and must be optimised for the comfort of physicians.

In developing the software, in cooperation with medical advisors, new limitations and standards emerged; this is the only way to consolidate a multidisciplinary project.

The quality of the images provided must have some standard sharpness and correct pigmentation to be processed by the application.

\section{Conflict of Interest}

The authors declare no conflict of interest.

\section{References}

[1] E. Poquioma. "Situación del cáncer de mama en el Perú". Presentation. Instituto Nacional de Enfermedades Neoplásicas (INEN), 2008.

[2] C. Ramos, D. Venegas. "Análisis de la Situación del Cáncer en el Perú". Presentation. Dirección General de Epidemiología, Ministerio de Salud del Perú, 2013.

[3] S. Coronato, G. Laguens, O. Spinelli, W. Di Girolamo. "Marcadores tumorales en cancer de mama" MEDICINA (Buenos Aires), 63, 73-82, 2002.

[4] B. Hirata, B. Karina, et al. "Molecular markers for breast cancer: prediction on tumor behavior." Disease markers, 2014. http://dx.doi.org/10.1155/2014/513158

[5] H. Xin et al. "Ki-67 is a valuable prognostic predictor of lymphoma but its utility varies in lymphoma subtypes: evidence from a systematic metaanalysis." BMC cancer, 14, 153, 2014. http://doi:10.1186/1471-2407-14-153

[6] S. Bustreo, S. Osella-Abate, et al. "Optimal Ki67 cut-off for luminal breast cancer prognostic evaluation: a large case series study with a long-term follow-up" Breast Cancer Res Treat, 157(2), 363-371, 2016. 10.1007/s10549-016-3817-9

[7] L. Fulawka, A. Halon. "Ki-67 evaluation in breast cancer: The daily diagnostic practice" Indian J. Pathol Microbiol, 60(2), 177-184, 2017. http://doi:10.4103/IJPM.IJPM 732_15

[8] Gudlaugsson, E., Skaland, I., Janssen, E. A., Smaaland, R., Shao, Z., Malpica, A., ... \& Baak, J. P. (2012). Comparison of the effect of different techniques for measurement of Ki67 proliferation on reproducibility and prognosis prediction accuracy in breast cancer. Histopathology, 61(6), 11341144.

[9] Zhong, F., Bi, R., Yu, B., Yang, F., Yang, W., \& Shui, R. (2016). A comparison of visual assessment and automated digital image analysis of Ki67 labeling index in breast cancer. PloS one, 11(2), e0150505.

[10] Koopman, T., Buikema, H. J., Hollema, H., de Bock, G. H., \& van der Vegt, B. (2018). Digital image analysis of Ki67 proliferation index in breast cancer using virtual dual staining on whole tissue sections: clinical validation and inter-platform agreement. Breast cancer research and treatment, 169(1), 3342.

[11] V. Anari, P. Mahzouni, R. Amirfattahi "Computer-aided Detection of Proliferative Cells and Mitosis Index in Inmunohistichemically Images of Meningioma" In 6th Iranian Conference on Machine Vision and Image Processing, Isfahan, Iran. IEEE, 2010. 10.1109/IranianMVIP.2010.5941151

[12] Grala, B., Markiewicz, T., Kozłowski, W., Osowski, S., Słodkowska, J., \& Papierz, W. (2009). New automated image analysis method for the assessment of Ki-67 labeling index in meningiomas. Folia Histochemica et Cytobiologica, 47(4), 587-592.

[13] M. Bouzid, A. Khalfallah, A. Bouchot, M. Selim, F. Marzani. "Automatic Cell Nuclei Detection: a Protocol to Acquire Multispectral Images and to compare results between colour and multispectral images" in SPIE Photonics West, BIOS, Imaging, Manipulation and Analysis of Biomolecules, Celles and Tissues XI, San Francisco, United States, 2013. doi.10.1117/12.2001980

[14] K. Ahmad, N. Yusoff. "Breast Cancer Types Based on Fine Needle Aspiration Biopsy -Data Using Random Forest Classifier" in I 13th International Conference on Intellient Systems Design and Applications, Bangi, Malaysia. IEEE, 2013. doi.10.1109/ISDA.2013.6920720

[15] R. Gonzalez, R. Woods. Digital Image Processing, 3rd. Ed., Prentice Hall, 2008.

[16] T. Chun-Ming, L. His-Jian. "Binarization of Colour Document Images via Luminance and Saturation Colour Features" IEEE Transactions on Image Processing, 11(4), 2002. 10.1109/TIP.2002.999677

[17] R. Gonzales, R. Woods, E. Eddins. Digital Imaging Processing Using Matlab, 2nd Ed., Mcgraw Hill, 2010.

[18] J.L Fleiss. "Measuring Nominal Scale Agreement Among Many Raters" Psycological Bulletin, 76(5): 378-382, 1971 http://dx.doi.org/10.1037/h0031619

[19] M. Sokolova, N. Japkowicz, S. Szpakowicz. "Beyond Accuracy, F-Score and ROC: A Family of Discriminant Measures for Performance Evaluation" In AI 2006: Advances in Artificial Intelligence, Lecture Notes in Computer Science (vol. 4304), 2006. DOI: 10.1007/11941439 114 